\title{
KN95 filtering facepiece respirators distributed in South Africa fail safety testing protocols
}

\author{
L Mottay, ${ }^{1 *} \mathrm{MB}$ ChB, FCP (SA), Cert Pulmonology (SA); J le Roux, ${ }^{1,4 \star}$ BSc Hons; \\ R Perumal, ${ }^{1}$ MB ChB, FCP (SA) Cert Pulmonology (SA), MPH, MMed; A Esmail, ${ }^{1}$ MD, FCP (SA), Cert Pulmonology (SA); \\ L Timm, ${ }^{4}$ BEngSc (BME), BScEng (Elec); S Sivarasu, ${ }^{4}$ PhD; K Dheda, ${ }^{1,2,3}$ MB BCh, FCP (SA), FCCP, PhD (Lond), FRCP (Lond)
}

${ }^{1}$ Centre for Lung Infection and Immunity, Division of Pulmonology and UCT Lung Institute, Department of Medicine, Faculty of Health Sciences, University of Cape Town, South Africa

${ }^{2}$ Institute of Infectious Diseases and Molecular Medicine, Faculty of Health Sciences, University of Cape Town, South Africa

${ }^{3}$ Faculty of Infectious and Tropical Diseases, London School of Hygiene and Tropical Medicine, London, UK

${ }^{4}$ Division of Biomedical Engineering, Department of Human Biology, Faculty of Health Sciences, University of Cape Town, South Africa

${ }^{*}$ Authors contributed equally

Corresponding author: KDheda (keertan.dheda@uct.ac.za)

Background. Given the global shortage of N95 filtering facepiece respirators (FFP2 in Europe) during the COVID-19 pandemic, KN95 masks (Chinese equivalent of the N95 and FFP2) were imported and distributed in South Africa (SA). However, there are hardly any published independent safety data on KN95 masks.

Objectives. To evaluate the seal, fit and filtration efficiency of several brands of KN95 masks marketed for widespread use in SA healthcare facilities, using standardised testing protocols.

Methods. The verifiability of manufacturer and technical details was first ascertained, followed by evaluation of the number of layers comprising the mask material. The testing protocol involved a directly observed positive and negative pressure user seal check, which if passed was followed by qualitative fit testing (sodium saccharin) in healthy laboratory or healthcare workers. Quantitative fit testing (3M) was used to validate the qualitative fit testing method. The filtration efficacy and integrity of the mask filter material were evaluated using a particle counter-based testing rig utilising aerosolised saline (expressed as filtration efficacy of $0.3 \mu \mathrm{m}$ particles). Halyard FLUIDSHIELD 3 N95 and 3M 1860 N95 masks were used as controls.

Results. Twelve KN95 mask brands (total of 36 masks) were evaluated in 7 participants. The mask type and manufacturing details were printed on only 2/12 brands (17\%) as per National Institute of Occupational Safety and Health and European Union regulatory requirements. There was considerable variability in the number of KN95 mask layers (between 3 and 6 layers in the 12 brands evaluated). The seal check pass rate was significantly lower in KN95 compared with N95 masks (1/36 (3\%) v. 12/12 (100\%); $p<0.0001)$. Modification of the KN95 ear-loop tension using head straps or staples, or improving the facial seal using Micropore $3 \mathrm{M}$ tape, enhanced seal test performance in 15/36 KN95 masks evaluated (42\%). However, none of these 15 passed downstream qualitative fit testing compared with the control N95 masks ( $0 / 15$ v. $12 / 12 ; p<0.0001)$. Only $4 / 8(50 \%)$ of the KN95 brands tested passed the minimum filtration requirements for an N95 mask (suboptimal KN95 filtration efficacy varied from $12 \%$ to $78 \%$, compared with 56\% for a surgical mask and $>99 \%$ for the N95 masks at the $0.3 \mu \mathrm{m}$ particle size).

Conclusions. The KN95 masks tested failed the stipulated safety thresholds associated with protection of healthcare workers against airborne pathogens such as SARS-CoV-2. These preliminary data have implications for the regulation of masks and their distribution to healthcare workers and facilities in SA.

S Afr Med J 2021;111(3):234-239. https://doi.org/10.7196/SAMJ.2021.v111i3.15381

The COVID-19 pandemic caused by SARS CoV-2 has claimed almost 1 million lives since its emergence approximately a year ago. ${ }^{[1]}$ Healthcare workers face a disproportionate burden of both morbidity and mortality as a result of occupational exposure, which may also result in nosocomial transmission of COVID-19 by healthcare workers and diminished capacity of healthcare systems. Indeed, 20\% of all cases of Middle East respiratory syndrome (MERS) and 20\% of all severe acute respiratory syndrome (SARS) cases globally occurred in healthcare workers, $10 \%$ of whom lost their lives. ${ }^{[2,3]}$ Healthcare workers are at least three times more likely than the general public to become infected with SARS-CoV-2, even after accounting for other risk factors and their greater access to testing. ${ }^{[4]}$

Infection in healthcare workers may be mitigated by a variety of strategies including triaging and administrative controls, environmental controls including good ventilation and ultraviolet germicidal irradiation, and use of personal protective measures and equipment, including protective masks. Use of filtering facepiece respirators (FFRs) has been shown to mitigate the SARS-CoV-2 infection risk in healthcare workers by as much as $86 \%$, especially if used during high-risk aerosol-generating procedures. ${ }^{[5,6]}$ However, there has been a worldwide shortage of high-quality, regulatoryapproved and authentic FFRs owing to a combination of factors including disruption of the global supply chain, increased demand, inequitable distribution, and unethical practices of hoarding, misuse, price gouging and export blocking. ${ }^{[7]}$ The N95 mask (FFP2 in Europe) has remained the primary mode of respiratory protection in most parts of the world because of the high regulatory standards to which it is manufactured. ${ }^{[8]}$ However, owing to the severe global shortage of 
N95 masks during the COVID-19 pandemic, there has been a growing demand for N95like FFRs. The most commonly available substitute for the N95 mask has been the KN95 mask, which is mainly manufactured in China to the GB2626-2006 standard, and which is considered equivalent to the N95 by the US Food And Drug Administration (FDA) following Emergency Use Authorisation based on testing of filtration efficacy of the mask material..$^{[8]}$

The manufacture of N95-like masks remains highly regulated in order to ensure that they filter out at least $95 \%$ of penetrating aerosol particles $\geq 0.3 \mu \mathrm{m}$ in diameter, fit tightly to maximise the passage of inhaled air through the filter fabric of the mask, and have low inhalational resistance to minimise breathing difficulty. There has been a growing concern about the quality of the circulating supplies of N95-like masks in many parts of the world, including the circulation of counterfeit masks.

\section{Objectives}

To evaluate the fit and safety of the KN95 mask against available N95 masks.

\section{Methods}

\section{Participants and setting (Fig. 1)}

We performed an observational study of user seal check and fit test pass rates for both N95 and KN95 respirators in 7 healthcare and laboratory workers employed at the Centre for Lung Infection and Immunity at the University of Cape Town, South Africa (SA). The 7 volunteers were screened for symptoms of COVID-19 prior to testing, which was conducted in a room with open windows and with the operator wearing an N95 mask. Standard precautions were followed, including hand sanitisation and cleaning of surfaces after testing. The 7 participants, all with prior experience of N95 respirator use, were of Indian $(n=3)$, Asian (Chinese, $n=1$ ), European ( $n=1)$, black African $(n=1)$ and mixed $(n=1)$ ethnicity. The study was approved by the Human Research Ethics Committee of the University of Cape Town (ref. no. 476/2020).

\section{Manufacturer details}

Twelve brands of KN95 masks were donated for testing by individuals, distributors, or doctors from different hospitals. Each sample was inspected for manufacturer details, which were then compared with the National Institute of Occupational Safety and Health (NIOSH) website (https://www. cdc.gov/niosh/npptl/topics/respirators/disp_ part/respsource.html), initially on receipt of the mask and thereafter by a second

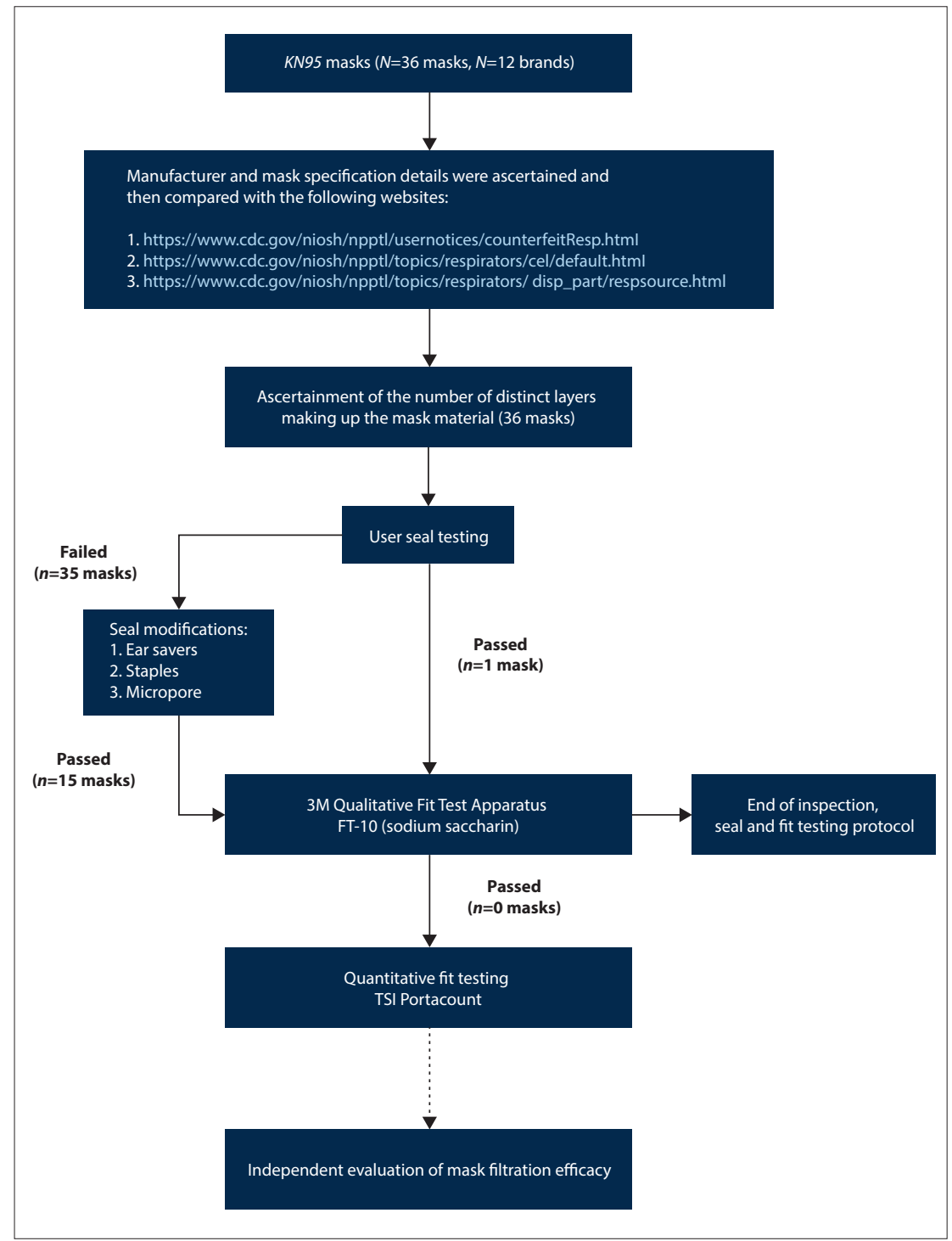

Fig. 1. Study outline, including the testing protocol used and the number of masks evaluated at each stage.

person prior to publication (last checked on 29 September 2020).

\section{Evaluation of mask layers}

Each mask was inspected, and the observations were recorded. Thereafter one mask from each brand was cut with a pair of scissors to inspect how many distinct layers the mask comprised.

\section{Control masks and tests of inward and outward leakage using the seal} test

The N95 respirator brand was selected as the control mask (FLUIDSHIELD 3 N95 Particulate Filter Respirator; Halyard Health, USA). Three masks from each of the 12 brands of KN95 respirators from various manufacturers were evaluated in participants. All participants performed a positive and negative pressure user seal check in accordance with the manufacturer's instructions under direct observation by a pulmonologist. A user seal check is a selfexamination to identify inward or outward leakage through visual and tactile detection of gaps and air leaks between the mask and the wearer's face. ${ }^{[9]}$ Briefly, to check for good fit, the wearer inhaled and exhaled several times to check whether the respirator collapsed slightly upon inhaling and expanded upon exhaling, and whether they could feel any air leaking past the respirator. If the mask failed the user seal test on the first attempt, we allowed two further attempts before making the determination of passing or failure.

\section{Modification of KN95 masks and tests of inward mask leakage using the fit test}

We also performed modifications to the mask ear loops with head straps (plastic strap with angulated prongs that attempted to improve facial seal by increasing ear- 
strap tension; UCT Hearo Ear Savers, http://www.rci.uct.ac.za/rcips/innovation_ achievements/products/hearo), staples or Micropore 3M tape. Masks that passed the user seal check were subjected to qualitative fit testing using the $3 \mathrm{M}$ Qualitative Fit Test Apparatus FT-10 (3M, USA), in accordance with the Occupational Safety and Health Administration Standard for Respiratory Protection (29 CFR 1910.134), which was administered by a pulmonologist. Participants with masks that passed a user seal check and a qualitative fit test were invited, according to the protocol, to do a quantitative fit test using a TSI PortaCount Respirator Fit Tester (model 8038; TSI, USA). For all testing stages (seal test, fit test, etc.) the Halyard FLUIDSHIELD N95 respirator was evaluated in parallel as a control. In addition, for the filtration efficacy testing, the Halyard N95, the 3M 1860 N95 Particulate Respirator (3M, USA) and nonsterile surgical masks were used as controls.

\section{Evaluation of filtration efficacy of the mask filter material}

Eight brands of KN95 masks underwent filtration integrity testing using a closed system (Fig. 2). Both the Halyard FLUIDSHIELD respirator and the 3M 1860 respirator were used as positive controls, and surgical masks were used as additional controls. Different mask samples were clampfixed within a cylindrical tube with an inner diameter of $85 \mathrm{~mm}$. Normal saline $(0.9 \%$ $\mathrm{NaCl}$ ) was aerosolised using a jet nebuliser (Prime Care Nebulizer Lot 190183; Prime Care, China) at a rate of $3 \mathrm{~L} / \mathrm{min}$. Airflow (high-efficiency particulate (HEPA) filtered) through the testing apparatus was generated using a HAILEA 328-ACO (HAILEA, China) air compressor, providing a combined flow through the closed system of $\sim 85 \mathrm{~L} / \mathrm{min}$. After passing through a drying chamber, the aerosolised particles were enumerated using a laser particle counter (Model 3886 GEO a; Kanomax, Japan). One-minute particle counts (of particle sizes $0.3 \mu \mathrm{m}, 0.5 \mu \mathrm{m}$, $1 \mu \mathrm{m}, 3 \mu \mathrm{m}$ and $5 \mu \mathrm{m}$ ) were conducted at the upstream and downstream probe locations (Fig. 2). The upstream and downstream counts were sampled from one region at one time. Each measurement was repeated three times. Single-pass filtration efficiency was measured as follows:

$$
\text { Filtration efficiency }(\%)=
$$

$\frac{\text { Upstream particle count }- \text { Downstream particle count }}{\text { Upstream particle count }} \times 100$

The average filtration efficiency was calculated from the three measurements conducted on each mask. Prior to each measurement, the flow velocity, humidity and temperature were measured by sampling air from the exhaust chamber. The mean (standard deviation (SD)) air temperature prior to each test was $23(2)^{\circ} \mathrm{C}$, with a relative humidity (RH) of $25(6) \% \cdot{ }^{[10,11]}$

\section{Data analysis}

All data were analysed using SPSS software 25.0 (IBM, USA). For all statistical comparisons, a $5 \%$ level of significance was used. A $\chi^{2}$ test was used to assess for differences in the performance of N95 and KN95 masks with regard to the evaluation of mask seal and fit. Descriptive statistics were used to present the mask filtration data.

\section{Results}

\section{Participants, mask identifiers and} mask layers

Seven healthy volunteers were included in the study; 6 (85\%) were female, and all were healthcare or laboratory workers with prior FFR experience. We selected 12 KN95 brands (total of 36 masks) from various manufacturers for testing (Table 1), with each brand tested in 3 participants. Of the 12 brands evaluated, only 8 brands were received in the original packaging, with 4 brands being received in clear plastic bags. Nevertheless, only 2 of the 12 brands had the mask type (i.e. KN95) and manufacturing details printed on each mask as per NIOSH and European Union regulatory requirements. ${ }^{[12,13]}$ All 12 KN95 brands had malleable metal nose strips and ear loops. One brand had three distinct layers, 6 brands had four layers, and 5 brands had five layers.

\section{Inward and outward leakage using the seal test}

Each participant tested a suitably sized N95 respirator and three KN95 respirators. In total, $12 \mathrm{~N} 95$ respirators and $36 \mathrm{KN} 95$ respirators were evaluated with a user seal check. Performance of the tested KN95 respirators was significantly poorer than the tested N95 respirators when evaluated using a user seal check $(1 / 36$ v. $12 / 12 ; p<0.0001)$.

\section{Effect of mask modification and qualitative fit testing}

Modification of the KN95 ear-loop tension using head straps or staples, or improving the facial seal using Micropore tape, enhanced

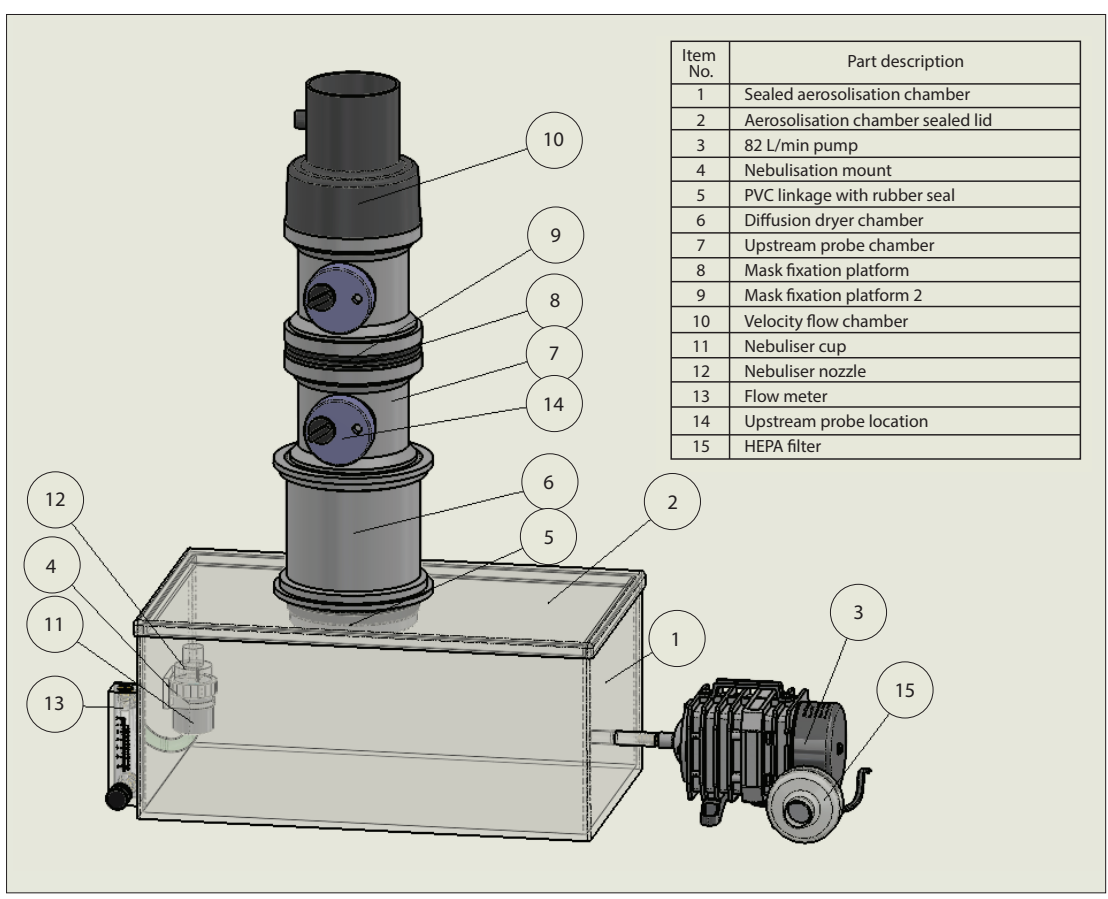

Fig. 2. Overview of the mask filtration efficiency testing system. The masks were fixed over a cylindrical duct with a inner diameter of $85 \mathrm{~mm}(8,9)$. The nebuliser cup (11) was filled with $0.9 \%$ saline solution. Pressurised air from a compressed air source was regulated to $3 \mathrm{~L} / \mathrm{min}$ using a flowmeter (13). Supplementary air was provided by an $82 \mathrm{~L} /$ min electromagnetic air pump - the total airflow through the closed system was therefore $85 \mathrm{~L} / \mathrm{min}$ (3) with a HEPA-filtered inlet (15). Aerosolised particles mixed with supplementary air within the arosolisation chamber (1) and passed through a diffusion drying chamber (6). Following a 30-second aerosolisation period, a series of three measurements was taken for 1 minute each at both the upstream (14) and downstream probing chambers using an optical particle counter (Kanomax Model 3886 GEO $\alpha$ ). Velocity flow, temperature and relative humidity were consistently measured using a probe within the exhaust duct. (HEPA = high-efficiency particulate.) 


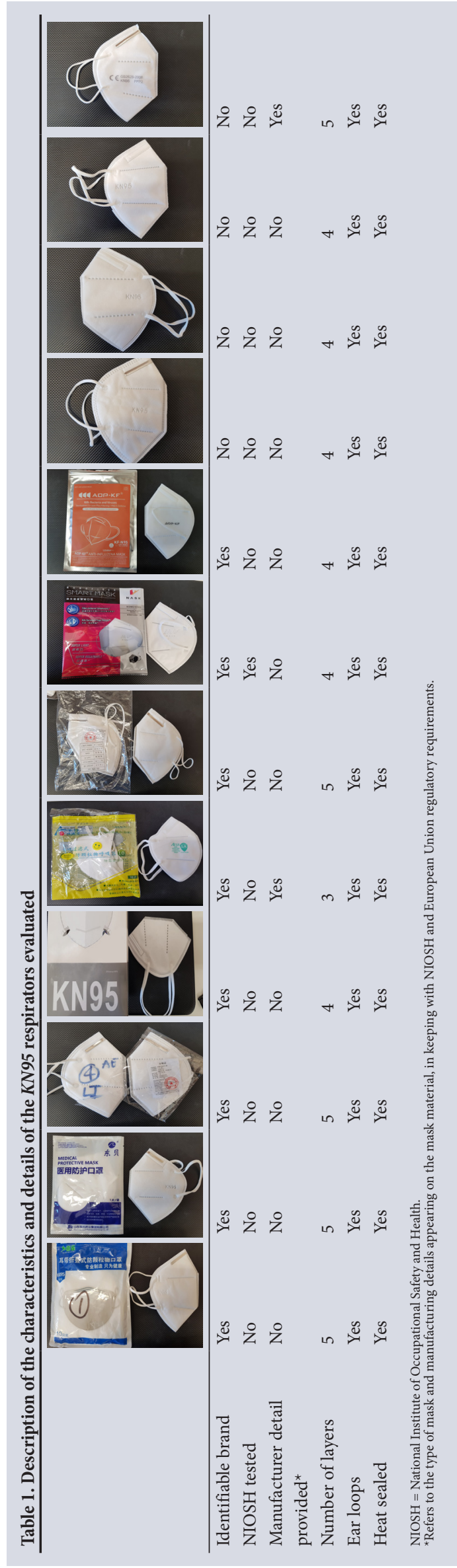

seal test performance in 15/36 KN95 masks evaluated (42\%). However, none of these 15 passed downstream qualitative fit testing compared with the control N95 masks (0/15 v. 12/12; $p<0.0001)$. The other 21/36 KN95 respirators did not pass a user seal check and could not be evaluated further by a qualitative fit test (Table 2).

\section{Filtration efficacy of the mask filter material}

Only the 2 positive control masks, as well as 4 of the 8 tested KN95 masks, performed as expected, filtering $>95 \%$ of all particles ranging from $0.3 \mu \mathrm{m}$ to $5 \mu \mathrm{m}$. Fifty percent of the KN95 masks evaluated failed to meet the minimum requirements of an N95 equivalent. Two of the KN95 masks tested demonstrated a filtration efficiency significantly less than that of the surgical masks tested (Fig. 3 and Table 3 ).

\section{Discussion}

Given the lack of available safety data, we conducted a preliminary study to evaluate 12 brands of KN95 masks (total of 36 masks) in 7 participants. Our major findings were that: $(i)$ of the 8 brands with identifiable manufacturer details, only 1 was on the NIOSH-approved list; (ii) the mask type and manufacturing details were printed on only $2 / 12$ (17\%) brands, as per NIOSH and European Union regulatory requirements; (iii) the number of individual mask layers in 12 different brands varied between 3 and 6; (iv) only 1 of the 36 KN95 masks passed the seal test; $(v)$ even after modification of the earloop tension using different methods, all 15 of the masks passing the seal test failed the qualitative fit testing; and ( $v i)$ of the 8 masks tested, only 4 passed filtration efficacy testing, i.e. had a filtration efficacy of $>95 \%$ for $0.3 \mu \mathrm{m}$ particles. In contrast, the N95 masks tested passed the seal test, qualitative fit testing, and mask filter integrity at the $0.3 \mu \mathrm{m}$ particle count size. In summary, none of the KN95 masks evaluated met the required safety criteria, as stipulated by the NIOSH and/or recently published by the South African Health Products Regulatory Authority (SAHPRA) ${ }^{[13-16]}$ to protect healthcare workers from dangerous aerosol-containing pathogens such as SARS-CoV-2 and/or Mycobacterium tuberculosis.

Almost all KN95 masks (35/36) failed the seal testing, and all the KN95 masks that were tested failed qualitative fit testing. Therefore, even before evaluating the integrity of the mask filter material, lack of an adequate facial seal was the major shortcoming. Previous studies have shown that inward facial leakage is the main mechanism by which FFRs fail testing. ${ }^{(9,17)}$ Interestingly, attempting to improve the facial seal by modifying the ear-loop tension failed to improve results (we evaluated several different methods, including increasing ear-loop tension by knotting, staples or head straps, or improving the facial seal using Micropore tape). To our knowledge, this is the first study that has attempted to objectively test modifications to improve facial seal and hence fit testing of KN95 masks. We conclude, based on our results, that mask design and the nature of the material used are major determinants of mask failure rather than the amount of tension that is required to hold the mask in place. Another determinant of inward mask leakage is facial structure. Indeed, Yu et al. ${ }^{[17]}$ showed that when a Chinese population was tested, only 2 out of 50 KN95 masks (4\%) passed leakage tests. Interestingly, in that study several N95 masks also had high inward leakage. The authors speculate that the mongoloid facial structure, especially at the bridge of the nose and chin level, facilitates inward facial leakage. The participants in our study were Asian, European, Indian, black African or of mixed race. In all the groups, the N95 control masks failed to show inward leakage.

Only 4 out of the 8 KN95 masks tested passed the mask filter integrity testing, i.e. were able to filter $>95 \%$ of particles $0.3 \mu \mathrm{m}$ in size. This filtration efficacy requirement is to ensure that organisms such as M. tuberculosis and viral particles complexed to respiratory secretions fail to penetrate the mask filter material. Some KN95 masks, although filtration integrity was of adequate standard, therefore still failed on facial seal and inward leakage of particles. Plana et al. ${ }^{[8]}$ (non-peer reviewed data in preprint format) also found that several KN95 masks failed to meet mask filter integrity thresholds. Their article highlighted that a high proportion of KN95 masks may be counterfeit. They suggest that the 
Table 2. Summary of results for tests of inward mask leakage

\begin{tabular}{|c|c|c|c|}
\hline & N95 masks $(N=12), n(\%)$ & KN95 masks $(N=36), n(\%)$ & $p$-value \\
\hline User seal check passed on first attempt & $12 / 12(100)$ & $1 / 12(3)$ & $<0.0001$ \\
\hline User seal check passed within 3 attempts after modification & $12 / 12(100)$ & $15 / 36(42)$ & $<0.05$ \\
\hline Qualitative fit test passed after modification of masks ${ }^{\star}$ & $12 / 12(100)$ & $0 / 15(0)$ & $<0.0001$ \\
\hline
\end{tabular}

Table 3. Face mask FE assessments using aerosolised sodium chloride particles

\begin{tabular}{|c|c|c|c|c|c|c|c|}
\hline \multirow[b]{2}{*}{ Mask name } & \multicolumn{5}{|c|}{ Single-pass filtration efficiency (\%), mean (SD) } & \multirow[b]{2}{*}{$\mathrm{T}\left({ }^{\circ} \mathrm{C}\right)$} & \multirow[b]{2}{*}{ RH (\%) } \\
\hline & $0.3 \mu \mathrm{m}$ & $0.5 \mu \mathrm{m}$ & $1 \mu \mathrm{m}$ & $3 \mu \mathrm{m}$ & $5 \mu \mathrm{m}$ & & \\
\hline 3M 1860 & $99.61(0.12)$ & $99.93(0.01)$ & $99.97(0)$ & $99.99(0.01)$ & $100(0)$ & 23.8 & 22.1 \\
\hline Halyard FLUIDSHIELD & $99.82(0.02)$ & $99.94(0.01)$ & $99.97(0.01)$ & $99.99(0.01)$ & $100(0)$ & 22.6 & 26.7 \\
\hline Surgical mask & $55.64(5.04)$ & $81.09(1.72)$ & $91.96(0.35)$ & $89.07(14.54)$ & $99.52(0.02)$ & 24.9 & 23.6 \\
\hline KN95 \#1 & $97.52(0.34)$ & $99.64(0.06)$ & $99.91(0.01)$ & $99.99(0.01)$ & $100(0)$ & 24.6 & 23.5 \\
\hline KN95 \#3 & $53.40(5.89)$ & $93.32(1.18)$ & $98.94(0.2)$ & $99.93(0.01)$ & $99.94(0.10)$ & 25.0 & 25.9 \\
\hline KN95 \#4 & 41.79 (3.6) & $80.66(1.73)$ & $94.41(0.53)$ & $99.20(0.02)$ & $99.94(0.11)$ & 24.6 & 17.6 \\
\hline KN95 \#5 & $99.12(0.17)$ & $99.85(0.03)$ & $99.95(0.01)$ & $100(0)$ & $100(0)$ & 22.9 & 20.3 \\
\hline KN95 \#7 & $96.63(0.51)$ & $99.44(0.10)$ & $99.87(0.03)$ & $99.99(0)$ & $100(0)$ & 23.7 & 19.6 \\
\hline KN95 \#8 & 78.49 (1.95) & $93.23(0.32)$ & $97.94(0.15)$ & $99.61(0.08)$ & $99.97(0.05)$ & 22.8 & 23.8 \\
\hline KN95 \#9 & $12.13(3.55)$ & $54.47(2.21)$ & $78.00(4.47)$ & $93.94(3.31)$ & $99.15(0.61)$ & 24.2 & 28.3 \\
\hline KN95 \#12 & $96.78(0.36)$ & $99.37(0.08)$ & $99.82(0.03)$ & $99.96(0.02)$ & $100(0)$ & 23.3 & 21.4 \\
\hline
\end{tabular}

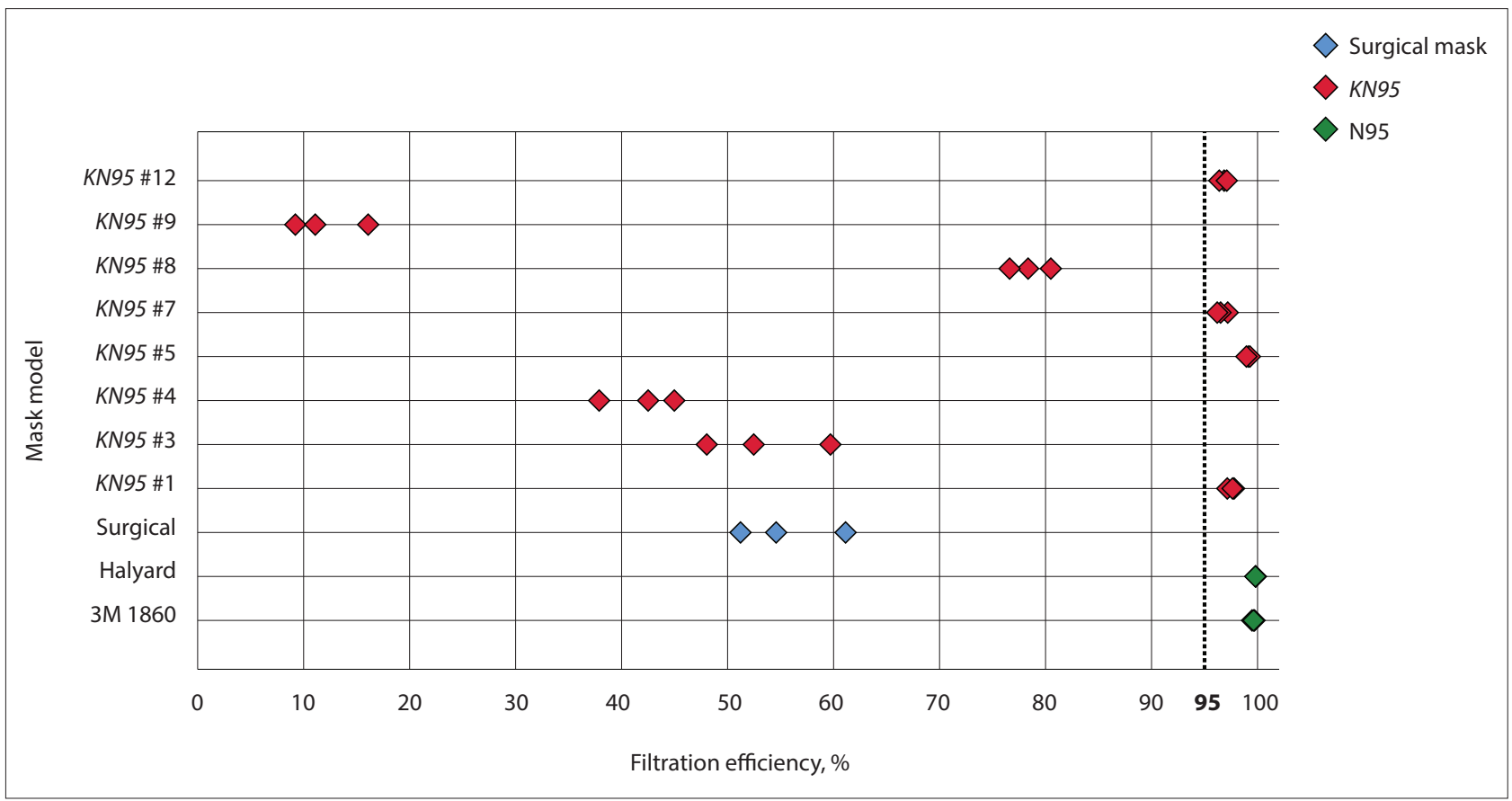

Fig. 3. Reproducibility of the filtration testing protocol, showing individual data points of the triplicate testing protocol. The filtration efficiency of each mask was tested in triplicate (Halyard and $3 M$ appear as single data points because of high reproducibility and overlapping data observations). The Halyard FLUIDSHIELD and $3 M 1860$ masks were used as positive controls, and a surgical mask was used as an additional control. Four brands of KN95 masks had filtration efficiency $<80 \%$, which was consistent over three filtration efficiency readings.

presence of ear loops, lack of proper labelling, lack of manufacturer source information, etc. all point to probable counterfeit origin. ${ }^{[15]}$

What are the implications of these findings? Our results are concerning because none of the KN95 masks tested met stipulated safety requirements, including passing the seal test and the qualitative fit test, and mask material filtration efficacy. We recommend stronger oversight by regulatory agencies such as SAHPRA to enforce recently published amended regulations and extend this to manufacturers and distributors. ${ }^{[18]}$ A discussion about access to regulatory testing such as is performed by the NIOSH (a US-based regulatory agency ${ }^{[15]}$ is warranted. However, it is noteworthy that testing is limited to manufacturer consistency and 
mask filtration efficacy, and not fit testing. ${ }^{[19]}$ We also recommend that fit testing be enforced, and that institutional capacity throughout the country be improved for qualitative fit and mask filter material efficacy testing. The former is an easy-to-perform, low-cost testing procedure, which can easily verify gross adequacy of mask safety. Specific institutions could therefore test KN95 masks at district or regional level before they are purchased from distributors. Regular in-house testing can also be performed to ensure consistency in batch quality and supply. We are currently exploring optimisation of a lowcost standardised mask filter integrity testing system. We would further recommend that the national pre-distribution evaluation protocols and regulations should include fit testing in a range of participants in additional to mask material filter integrity testing. Indeed, in the single NIOSH-approved KN95 mask that we evaluated, the filtration testing requirements but not the fit testing ones were met. It is important to note that $\sim 10-20 \%$ of masks passing qualitative fit testing may still fail quantitative fit testing, which is a more sensitive test. ${ }^{[20,21]}$ It would be useful to have a local website (perhaps under the auspices of a regulatory agency) that can vet masks that have already been tested and are deemed to be safe for use. Existing organisations and websites that publish tested and approved KN95 masks include the NIOSH Certified Equipment List or the NIOSH Trusted Source, and the FDA Appendix A or Exhibit $1{ }^{[21]}$ The Chinese regulatory agency for medical devices also provides details about National Medical Products Administration certification. ${ }^{[22]}$ Such sites also highlight clues to counterfeit masks that do not meet safety requirements, including lack of manufacturer and distributor information, the presence of ear loops, perforation in the mask material, lack of labelling on the mask certifying that it is approved, and embossing on $\mathrm{KN} 95$ s that exposes the underlying filter layer. ${ }^{[8]}$

\section{Study strengths and limitations}

The key strengths of our study include a testing protocol incorporating seal and fit testing and measuring the filtration integrity of the masks. To our knowledge, this is the only published study that has evaluated all these parameters and dimensions across a range of different KN95 brands and involving 36 different KN95 masks. However, the study has several limitations. We sampled only a limited number of masks, and only those accessible to us. However, some masks that were sent to us came from outside the Western Cape area, although most were already being used in hospitals in the Western Cape or in other regions of SA. Secondly, we only performed testing in a limited number of participants. Nevertheless, we conducted several different types of testing and included modifications to try to improve the facial seal, and further increasing participant sample size is unlikely to have changed our results. Thirdly, we did not measure and categorise facial dimensions. It would have been useful to test more participants of different racial backgrounds and with different facial structures. Finally, although we tested filter integrity of the masks, this may not equate to the type of testing using commercially standardised equipment that may be performed by certified bodies such as the NIOSH (and is offered by at least one commercial company in SA). Nevertheless, we did use similar flow rates (validated with a flow meter), a commercially available particle counter, a surgical mask as a negative control, and certified N95 masks as positive controls to validate our findings. We are in the process of validating our low-cost testing rig against a standardised commercially available system such as the TSI 8130A Automated Filter Tester (TSI, USA).

\section{Conclusions}

None of the 12 brands of KN95 masks tested (comprising 36 masks) met stipulated safety requirements known to prevent infection by dangerous respiratory pathogens including M. tuberculosis and
SARS-CoV-2. The findings presented here have implications for KN95 mask evaluation at both regulatory and institutional levels. Our findings will have relevance even after the COVID-19 epidemic has passed, because existing KN95 stockpiles may be given to healthcare workers to prevent infection by M. tuberculosis. We would recommend against this practice, given the failure of these masks to meet stipulated safety requirements.

Declaration. The research for this study was done in partial fulfilment of the requirements for JlR's $\mathrm{PhD}$ in Biomedical Engineering degree at the University of Cape Town.

Acknowledgements. The KD lab acknowledges funding from the SA MRC (RFA-EMU-02-2017), EDCTP (TMA-2015SF-1043, TMA-1051-TESAII, TMA-CDF2015), UK Medical Research Council (MR/S03563X/1) and the Wellcome Trust (MR/S027777/1).

Author contributions. KD conceived the idea and designed the study. LM and JIR collected the data. All the authors contributed to planning, logistics, data analysis and drafting of the manuscript.

Funding. South African Medical Research Council (RFA-EMU-02-2017). Conflicts of interest. None.

. World Health Organization. Coronavirus disease (COVID-19) weekly epidemiological update and weekly operational update. https://www.who.int/emergencies/diseases/novel-coronavirus-2019/ intuation-reports (accessed 20 July 2020)

World Health Organization. Summary of probable SARS cases with onset of illness from 1 November 2002 to 31 July 2003. 24 July 2015. https://www.who.int/publications/m/item/summary-of-probable sars-cases-with-onset-of-illness-from-1-november-2002-to-31-july-2003 (accessed 20 July 2020).
Xiao J, Fang M, Chen O He B. SARS, MERS and COVID-19 among healthcare workers: A narrative Xiao J, Fang M, Chen Q, He B. SARS, MERS and COVID-19 among healthcare workers: A narrative
review. J Infect Public Health 2020;13(6):843-848. https://doi.org/10.1016/j.jiph.2020.05.019 review. Infect Public Health 2020;13(6):843-848. https://doi.org/10.1016/j.jiph.2020.05.019
Nguyen LH, Drew DA, Graham MS, et al. Risk of COVID-19 among front-line health-care Nguyen LH, Drew DA, Graham MS, et al. Risk of COVID-19 among front-line health-care workers
and the general community: A prospective cohort study. Lancet Public Health 2020;5(9):e475-e483. and the general community: A prospective coh
https://doi.org/10.1016/S2468-2667(20)30164-X

5. Chu DK, Akl EA, Duda S, et al. Physical distancing, face masks, and eye protection to prevent personto-person transmission of SARS-CoV-2 and COVID-19: A systematic review and meta-analysis. Lancet 2020;395(10242):1973-1987. https://doi.org/10.1016/S0140-6736(20)31142-9

6. Iannone P, Castellini G, Coclite D, et al. The need of health policy perspective to protect healthcare workers during COVID-19 pandemic: A GRADE rapid review on the N95 respirators effectiveness. PLoS ONE 2020;15(6):e0234025. https://doi.org/10.1371/journal.pone.023402

Chersich MF, Gray G, Fairlie L, et al. COVID-19 in Africa: Care and protection for frontline healthcare workers. Global Health 2020;16(1):46. https://doi.org/10.1186/s12992-020-00574-3

8. Plana D, Tian E, Cramer AK, et al. Assessing the quality of nontraditional N95 filtering face-piece respirators available during the COVID-19 pandemic. medRxiv 2020 (epub 27 July 2020). https://doi. org/10.1101/2020.07.25.20161968

Krah J, Shamblin M, Shaffer R. Filtering out confusion: Frequently asked questions about respiratory protection, user seal check. National Institute for Occupational Safety and Health, April 2018. https:// www.cdc.gov S. Miller A, Eimer BC. Evalut.tinl (accessed 30 May 2020)

. Rengasamy S, Miller A, Eimer BC. Evaluation of the filtration performance of NIOSH-approved N95 filtering facepiece respirators by photometric and number-based test methods. J Occ Environ Hyg 2010;8(1):23-30. https://doi.org/10.1080/15459624.2010.515556

. National Personal Protective Technology Laboratory, National Institute for Occupational Safety and Health, Centers for Disease Control and Prevention. Assessment of filter penetration performance for
non-NIOSH approved respirators: NPPTL assessment to support the Covid-19 response. 31 March non-NIOSH approved respirators: NPPTL assessment to support the Covid-19 response. 31 March 2020. https://www.cdc.
(accessed 30 May 2020)

12. European Commission Directorate-General for Health and Food Safety. Guidance on regulatory requirements for medical face masks. 2020. https://ec.europa.eu/health/sites/health/files/md_sector/ docs/md_guidance-reg-req-med-face-masks.pdf (accessed 10 August 2020).

13. Centers for Disease Control and Prevention. NIOSH-approved particulate filtering facepiece respirators. Last reviewed 9 April 2020. https://www.cdc.gov/niosh/npptl/topics/respirators/disp_part/ default.html (accessed 3 October 2020).

14. Centers for Disease Control and Prevention. Transmission-based precautions. Last reviewed 7 January 2016. https://www.cdc.gov/infectioncontrol/basics/transmission-based-precautions.html (accessed 30 May 2020).

15. Centers for Disease Control and Prevention. Counterfeit respirators/misrepresentation of NIOSHapproval. https://www.cdc.gov/niosh/npptl/usernotices/counterfeitResp.html (accessed 3 October

16. Centers for Disease Control and Prevention. Certified equipment list. Last reviewed 9 April 2020. https://www.cdc.gov/niosh/npptl/topics/respirators/cel/default.html (accessed 3 October 2020).

77. Yu Y, Jiang L, Zhuang Z, et al. Fitting characteristics of N95 filtering-facepiece respirators used widely in China. PLoS ONE 2014;9(1):e85299. https://doi.org/10.1371/journal.pone.0085299

South African Health Products Regulatory Authority. MD025: Licensing and regulatory requirements for the manufacture and distribution of medical and respirator masks during Covid-19.8 September 2020. https://www.sahpra.org.za/wp-content/uploads/2020/09/MD025_Alternative-licensing-andregulatory-pathway-for-masks_September2020_vF.pdf (accessed 6 October 2020) US Food and Drug Administration. Stakeholders for non-NIOSH-approved imported FFRs manufactured in China. https://www.fda.gov/media/136664/download (accessed 6 October 2020).
Hon CY, Danyluk Q Bryce E, et al. Comparison of qualitative and quantitative fit-testing results for Hon CY, Danyluk Q, Bryce E, et al. Comparison of qualitative and quantitative fit-testing results for
three commonly used respirators in the healthcare sector. J Occup Environ Hyg 2017;14(3):175-179. three commonly used respirators in the healthc
https://doi.org/10.1080/15459624.2016.1237030

21. Danyluk Q, Hon CY, Neudorf M, et al. Health care workers and respiratory protection: Is the user seal check a surrogate for respirator fit-testing? J Occup Environ Hyg 2011;8(5):267-270. https://doi.org/ $10.1080 / 15459624.2011 .566016$

22. EMERGO. China NMPA medical device regulations. http://english.nmpa.gov.cn/ (accessed 30 May 2020). 